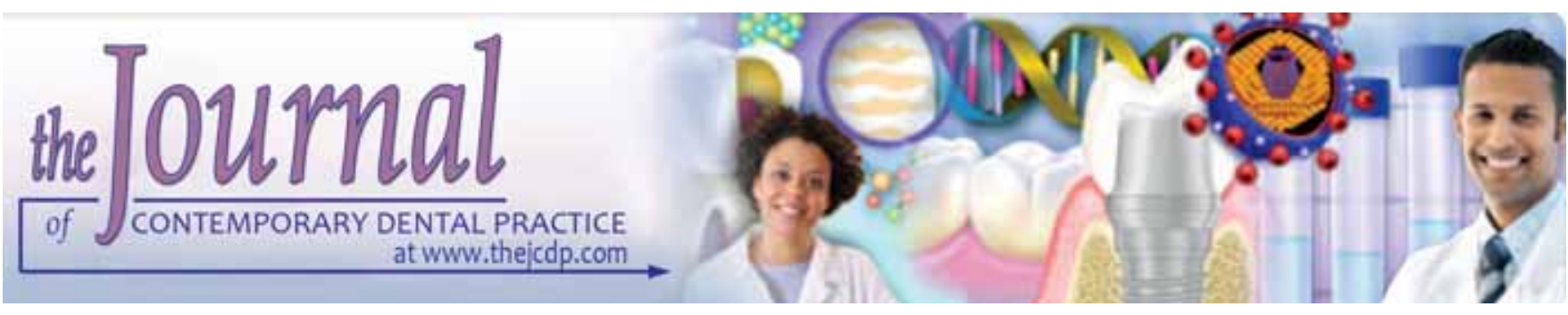

\title{
Influence of the Fast-processing Technique on the Number of the Occlusal Contacts and Occlusal Vertical Dimension of Complete Dentures
}

Parsa Atashrazm, Leila Zamani Alavijeh, Maryam Sadat Sadrzadeh Afshar

\section{ABSTRACT}

Aim: Occlusal errors during acryl processing affect the retention and stability of complete dentures. The aim of the present study was to assess the influence of a short curing technique on the number of occlusal contacts and the occlusal vertical dimension (OVD) of complete dentures.

Materials and methods: Complete dentures were prepared. The number of occlusal contacts was recorded in centric relation (CR) using $60 \mu$ articulation paper. The OVD was recorded with the waxed trial denture in place. Dentures were then invested and processed with compression molding and short cure water bath technique. The number of occlusal contacts was recorded again. The amount of pin opening was measured for all of the complete dentures on the articulator. Data were analyzed with paired t-test to determine the alterations.

Results: The mean number of occlusal contacts before and after processing was $10.9 \pm 2.4$ and $6.3 \pm 3.1$ respectively $(4.7 \pm$ 1.9 decrease; $p<0.001)$. A $2 \mathrm{~mm}$ mean increase in OVD was observed in $47.7 \%$ of the dentures with $<6$ occlusal contact changes and $88.9 \%$ of the dentures with $\geq 6$ occlusal contact changes $(p<0.003)$. A significant change in the number of occlusal contacts was associated with an OVD increased up to two times.

Conclusion: The short curing technique seems to be related to the decreased occlusal contacts and increased OVD.

Clinical significances: More time is needed to adjust the occlusal errors of this method, because it has a negative effect on the morphologic pattern of artificial teeth of complete dentures and thus should be used carefully.

Keywords: Complete denture, Compression molding, Fastprocessing technique, Occlusal contacts, Occlusal vertical dimension, Laboratory research.

How to cite this article: Atashrazm P, Alavijeh LZ, Afshar MSS. Influence of the Fast-processing Technique on the Number of the Occlusal Contacts and Occlusal vertical Dimension of Complete Dentures. J Contemp Dent Pract 2011;12(2):84-90.
Source of support: Nil

Conflict of interest: None declared

\section{INTRODUCTION}

Acrylic resin processing is usually associated with a number of occlusal errors, such as increased occlusal vertical dimension (OVD), changes in the number of expected occlusal contacts and occlusal plane alterations. ${ }^{1-5}$ The stability of a complete denture is highly dependent on an even distribution of the occlusal load, which can be achieved by obtaining the maximum intercuspation in the centric relation and by elimination of occlusal errors. ${ }^{6-9}$ This intercuspation should be established bilaterally on the posterior artificial teeth. Unilateral and insufficient occlusal contacts, which are defined as an incorrect occlusion, and the consequent trauma affect the retention and resistance; cause soft tissue soreness, accelerate residual ridge resorption; and decrease masticatory efficiency. ${ }^{1,10-14}$ Clinical and laboratory remount has been successfully used to locate the occlusal errors, which are then selectively eliminated to establish maximum intercuspation. ${ }^{15-19}$ A high number of errors may be time-consuming to correct and may also result in morphologic impairment of the artificial teeth. $^{7,8}$

Occlusal errors usually occur during mounting, acrylic processing, deflasking, finishing and polishing of the complete denture. ${ }^{1}$ Processing of the complete denture is composed of 43 steps. ${ }^{20}$ Compression molding, injection molding and the fluid resin technique are the most commonly used molding methods. The hot water bath (delayed, relatively delayed and fast), microwave and dry 
oven are used for the processing techniques. Compression molding is the most common laboratory molding method due to the simple equipment needed..$^{20,21}$

Several studies have discussed the effect of molding and processing methods on the OVD of the complete dentures. ${ }^{2-8}$ However, the effect of the applied method on the number and distribution of the occlusal contacts has not been investigated in great detail. ${ }^{3}$ Moreover, these studies have mostly been done on low numbers of simulated edentulous jaws or models. In addition, the hot water bath, the most frequently used method, has not been assessed in terms of changes in OVD in vitro.

The present study was conducted to assess the effect of compression molding, and the fast water bath processing technique on the number of the occlusal contacts and on the changes in OVD of complete dentures fabricated in the Department of Removable Prosthodontics, School of Dental Sciences, Islamic Azad University of Tehran during 2010. The null hypothesis of the present study stated that the compression molding and fast water bath processing techniques do not change the OVD and/or the number of occlusal contacts.

\section{MATERIALS AND METHODS}

This study was performed on 62 conventional complete dentures and included completely edentulous patients; master casts on which the teeth were setup. Anatomic acrylic teeth (Polident dd, 5293 Volcja Draga Slovenia) were used. The complete dentures were tried in to verify the maxillomandibular relations and for esthetic considerations. The complete dentures were wax contoured, and the number of occlusal contacts was then recorded using a $60 \mu$ articulating paper (Bausch Articulating Papers, Nashua, NH, USA). The articulating paper was cut according to the width of the premolars' and molars' cusps. The incisal pin was

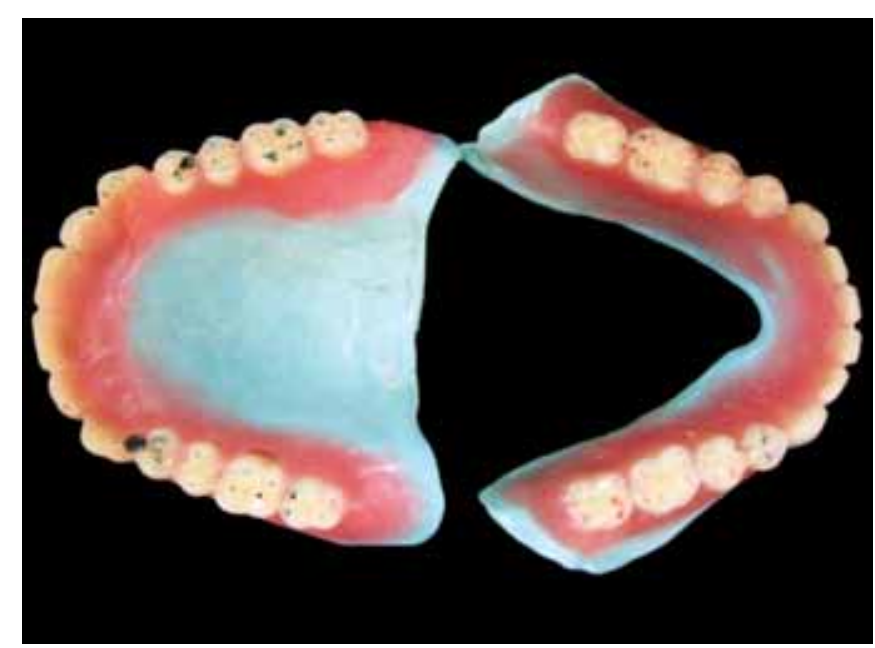

Fig. 1: Occlusal contacts (blue and red marks) of the studied complete dentures before processing removed from contacts with the incisal table. Then the occlusal contacts were separately recorded for the premolars and molars (Fig. 1). ${ }^{22}$ The OVD was recorded for all specimens according to the calibration of the registration groove of the incisal pin of the articulator (Haunu $\mathrm{H}_{2}$ Waterpick, Technologies Inc, Fort Collins, Co, USA), which was set at zero for all mounted complete dentures.

The casts were then cautiously removed from the mounting stone. Artificial teeth and the wax surface were washed with cold water and soap using soft cotton to wipe off the oil. Packing of the complete dentures was done according to standard protocols ${ }^{1}$ as follows. Artificial stone (Parsdandan, Tehran, Iran) was mixed with tap water and poured into the inferior half of the flask on a vibrator. The cast was then placed in the flask, and the stone was leveled between the edge of the cast and the rim of the flask. After separating the medium applied to the exposed stone in the flask, a core of artificial stone, that was 2 to $4 \mathrm{~mm}$ in thickness, was developed around the labial and buccal surfaces of both wax dentures on the lingual surface of the lower wax denture and the palatal surface of the upper wax denture. The top half of the flask was placed in position and the two flask halves met exactly. A mix of artificial stone was poured up to the level of the incisal edges of the anterior teeth and to the tips of the cusps of the posterior teeth. The exposed stone was painted with separating medium, the flask was completely filled with artificial stone and the lid of the flask was placed in position. The flask was placed in boiling water for 4 to 6 minutes based on the size of the flask. The flask was removed from the water and opened from the side opposite the greatest undercut of the cast. After the flask was opened, the surplus wax was washed out with a stream of boiling water. When the water had been drained from the flask, the mold was washed again with boiling water containing a detergent and then again with clean boiling water. After the stone was dry, but still hot, the inside of the mold and the cast were painted with a tinfoil substitute (MR Dental LTD, England) using a hair brush. The tinfoil substitute was allowed to dry and a second coat was painted on the inside of the mold. The flask was allowed to cool to room temperature.

Acrylic resin dough was made with a mixture of the powder (polymer) and liquid (monomer), (Selectaplus/ Trevalon Dentsply LTD, England) in accordance with the manufacturer's directions. When the mixture reached a dough consistency, it was packed in the mold (compression molding technique) and the flask was closed in a press with a sheet of separating plastic between the two halves until they were almost in approximation. The packing procedure was repeated until the mold was filled and no flash was formed. Next, the flask was closed completely without the 
separating sheet, and then the flask and clamp were placed in a curing unit, containing cold water. The flasks were heated gradually up to boiling temperature and left for 25 to 30 minutes at this temperature (fast-processing technique). Then the flask was allowed to cool to room temperature.

Deflasking was completed and the processed dentures were left on the casts. The casts and dentures were fitted back on to the mountings on the articulator and the processing changes were observed. The number of occlusal contacts was checked with articulating paper (Fig. 2) and the amount of pin opening that occurred secondary to processing was measured on the registration groove of the incisal pin of the articulator. The obtained data were analyzed using paired t-test. The coefficient of variation (CV) of the occlusal contacts was also studied.

\section{RESULTS}

The present study included 62 underconstruction complete dentures. Males and females composed 66.1 and 33.9\% of the edentulous patients, respectively. The patients ranged in age from 31 to 86 years (an average of $55 \pm 11.2$ years). A total of $58 \%$ of the patients were receiving a complete denture for the first time. The distribution of the dentures according to the number of occlusal contacts is shown in Table 1 . The mean number of occlusal contacts was $10.9 \pm$ 2.4 prior to processing and $6.3 \pm 3.1$ afterward. The coefficient of variations of the complete dentures according to the percent of occlusal contact changes before and after processing occlusal contacts was 22 and $47.6 \%$ respectively. After processing, bilateral and unilateral occlusal contacts were detected in 87.6 and $12.4 \%$ of the cases respectively. The occlusal contacts showed an average of $4.7 \pm 1.9$ decreases due to processing, which was statistically significant based on a paired t-test $(\mathrm{p}<0.001)$. The mean number of occlusal contacts in the complete dentures before and after processing is presented in

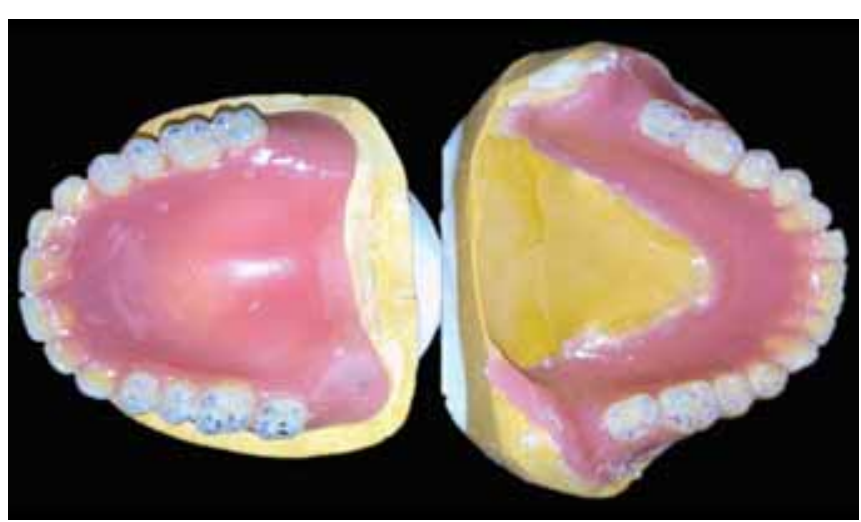

Fig. 2: The number of occlusal contacts (blue and red marks) of the studied complete dentures has significantly decreased in the same denture of figure 1 after processing
Table 2, and the distribution of the complete dentures based on the percent of occlusal contact changes before and after processing is shown in Fig. 3.

The OVD was changed by $1.9 \pm 0.4 \mathrm{~mm}$ after processing. The correlation between the changes in the number of occlusal contacts (more or less than 6) and the changes in the OVD alterations are shown in Table 3 . The mean number of occlusal contacts in the $<6$ occlusal contacts changes group and in the $\geq 6$ occlusal contacts changes group was $3.7 \pm 1$ and $7.2 \pm 1.4$ respectively. An OVD increase of $2 \mathrm{~mm}$ was seen in 47.7 and $88.9 \%$ of the $<6$ occlusal contact changes group and the $\geq 6$ occlusal contacts changes group respectively, which was statistically significant $(\mathrm{p}<0.003)$. A significant change in the number of occlusal contacts may be associated with an OVD increased up to two times $(\mathrm{RA}=2)$.

\section{DISCUSSION}

The present study showed that the mean number of occlusal contacts significantly decreases with processing. A significant relationship was found between the decreased number of occlusal contacts and increased OVD after processing. Dentures with a higher loss in the number of occlusal contacts showed increased OVD after processing.

The OVD has been investigated in similar studies. .,6,8,9 $^{2}$ Keenan et $\mathrm{al}^{4}$ measured intermolar width addition to assessing the OVD. Nogueira et $\mathrm{al}^{7}$ studied the OVD and dimensional changes. Campos et $\mathrm{al}^{3}$ assessed the number of occlusal contacts and the vertical height. Walter and Boening ${ }^{14}$ studied the proportion of right to left occlusal contacts. The number of occlusal contacts, which is an important factor in the stability of prostheses, and the vertical height alterations were investigated herein.

Most studies have used models prepared with standard molds; ${ }^{2,4,6-9}$ some have merely concerned a single jaw, ${ }^{4,6}$ while others have considered both jaws. ${ }^{2,7-9,14,15}$ The present study has included completely edentulous patients master casts on which teeth were setup. While models with standard molds have a uniform thickness of flanges in all samples and the same OVD, which are advantageous in most studies, master casts of the patients (as used in the present study) inevitably have no uniform thickness in terms of the dimensions of flanges and different OVD which may have an impact on the changes of the OVDs and the number of occlusal contacts. However, these patients are clinical cases that dentists may encounter in everyday practice; therefore, dental practitioners should consider the impact of the fast processing technique on the OVD and the number of occlusal contacts within complete dentures. In other words, this research is more near to the clinical practice than standard molds. 


\begin{tabular}{|c|c|c|c|c|c|c|}
\hline $\begin{array}{l}\text { Studied } \\
\text { complete } \\
\text { dentures }\end{array}$ & $\begin{array}{c}\text { Number of } \\
\text { occlusal contacts } \\
\text { before processing }\end{array}$ & $\begin{array}{l}\text { Number of } \\
\text { occlusal contacts } \\
\text { after processing }\end{array}$ & $\begin{array}{l}\text { Changes of } \\
\text { occlusal } \\
\text { contacts }\end{array}$ & $\begin{array}{c}\text { Occlusal vertical } \\
\text { dimension before } \\
\text { processing }\end{array}$ & $\begin{array}{l}\text { Occlusal vertical } \\
\text { dimension } \\
\text { after processing }\end{array}$ & $\begin{array}{l}\text { Changes of } \\
\text { occlusal vertical } \\
\text { dimension }\end{array}$ \\
\hline 1 & 8 & 4 & -4 & 0 & 2 & +2 \\
\hline 2 & 9 & 2 & -7 & 0 & 3 & +3 \\
\hline 3 & 10 & 3 & -7 & 0 & 2.5 & +2.5 \\
\hline 4 & 9 & 3 & -6 & 0 & 2.5 & +2.5 \\
\hline 5 & 10 & 8 & -2 & 0 & 2 & +2 \\
\hline 6 & 12 & 3 & $-\overline{9}$ & 0 & 3 & +3 \\
\hline 7 & 9 & 3 & -6 & 0 & 2 & +2 \\
\hline 8 & 11 & 3 & -8 & 0 & 3 & +3 \\
\hline 9 & 10 & 2 & -8 & 0 & 2 & +2 \\
\hline 10 & 12 & 1 & -11 & 0 & 4 & +4 \\
\hline 11 & 12 & 6 & -6 & 0 & 2 & +2 \\
\hline 12 & 10 & 8 & -2 & 0 & 1 & +1 \\
\hline 13 & 10 & 4 & -6 & 0 & 2 & +2 \\
\hline 14 & 11 & 6 & -5 & 0 & 2 & +2 \\
\hline 15 & 9 & 3 & -6 & 0 & 3 & +3 \\
\hline 16 & 9 & 4 & -5 & 0 & 2 & +2 \\
\hline 17 & 10 & 5 & -5 & 0 & 3 & +3 \\
\hline 18 & 9 & 6 & -3 & 0 & 3 & +3 \\
\hline 19 & 10 & 4 & -6 & 0 & 2 & +2 \\
\hline 20 & 11 & 7 & -4 & 0 & 2 & +2 \\
\hline 21 & 12 & 8 & -4 & 0 & 1.5 & +1.5 \\
\hline 22 & 10 & 3 & -7 & 0 & 2.5 & +2.5 \\
\hline 23 & 8 & 4 & -4 & 0 & 2 & +2 \\
\hline 24 & 8 & 4 & -4 & 0 & 2 & +2 \\
\hline 25 & 12 & 9 & -3 & 0 & 1 & +1 \\
\hline 26 & 8 & 4 & -4 & 0 & 1 & +1 \\
\hline 27 & 10 & 1 & -9 & 0 & 4 & +4 \\
\hline 28 & 10 & 3 & -7 & 0 & 4 & +4 \\
\hline 29 & 8 & 3 & -5 & 0 & 2 & +2 \\
\hline 30 & 8 & 4 & -4 & 0 & 2 & +2 \\
\hline 31 & 10 & 2 & -8 & 0 & 2 & +2 \\
\hline 32 & 12 & 11 & -1 & 0 & 1.5 & +1.5 \\
\hline 33 & 10 & 6 & -4 & 0 & 2 & +2 \\
\hline 34 & 8 & 7 & -1 & 0 & 3 & +3 \\
\hline 35 & 11 & 8 & -3 & 0 & 1 & +1 \\
\hline 36 & 14 & 9 & -5 & 0 & 2 & +2 \\
\hline 37 & 11 & 8 & -3 & 0 & $1 . \overline{5}$ & $+1 . \overline{5}$ \\
\hline 38 & 13 & 10 & -3 & 0 & 1 & +1 \\
\hline 39 & 17 & 13 & -4 & 0 & 1 & +1 \\
\hline 40 & 10 & 7 & -3 & 0 & 1 & +1 \\
\hline 41 & 12 & 9 & -3 & 0 & 0.5 & +0.5 \\
\hline 42 & 18 & 14 & -4 & 0 & 1 & +1 \\
\hline 43 & 16 & 11 & -5 & 0 & 2 & +2 \\
\hline 44 & 12 & 7 & -5 & 0 & 2 & +2 \\
\hline 45 & 15 & 11 & -4 & 0 & 1.5 & +1.5 \\
\hline 46 & 15 & 12 & -3 & 0 & 1 & +1 \\
\hline 47 & 14 & 8 & -6 & 0 & 1.5 & +1.5 \\
\hline 48 & 13 & 9 & -4 & 0 & 1.5 & +1.5 \\
\hline 49 & 12 & 8 & -4 & 0 & 2 & +2 \\
\hline 50 & 16 & 13 & -3 & 0 & 2 & +2 \\
\hline 51 & 11 & 6 & -5 & 0 & 0.5 & +0.5 \\
\hline 52 & 11 & 7 & -4 & 0 & 1 & +1 \\
\hline 53 & 8 & 4 & -4 & 0 & 2 & +2 \\
\hline 54 & 11 & 8 & -3 & 0 & $1 . \overline{5}$ & +1.5 \\
\hline 55 & 12 & 8 & -4 & 0 & 1 & +1 \\
\hline 56 & 11 & 7 & -4 & 0 & 1 & +1 \\
\hline 57 & 10 & 7 & -3 & 0 & 1 & +1 \\
\hline 58 & 13 & 9 & -4 & 0 & 1 & +1 \\
\hline 59 & 8 & 4 & -4 & 0 & 2 & +2 \\
\hline 60 & 8 & 4 & -4 & 0 & 2 & +2 \\
\hline 61 & 10 & 3 & -7 & 0 & 1.5 & +1.5 \\
\hline 62 & 8 & 5 & -3 & 0 & 1.5 & +1.5 \\
\hline
\end{tabular}


The tooth type has been similar in some studies. ${ }^{2-5,7-14,17}$ Anatomic acrylic teeth (Polident dd, 5293 Volcja Draga Slovenia) were used in the present study. Basso et $\mathrm{al}^{2}$ used balanced occlusion and lingualized occlusion in their study. Keenan et al ${ }^{4}$ and also Nogueira et $\mathrm{al}^{7}$ used lingualized occlusion. Walter and Boening ${ }^{14}$ studied bilateral balanced occlusion and Swards et $\mathrm{al}^{6}$ investigated the monoplane occlusal pattern. However, the occlusion type has not been mentioned in some studies. ${ }^{8,9}$ Bilateral balanced occlusion, the most common occlusion for the fabrication of complete removable dentures, was applied in the present study.

Keenan et $\mathrm{al}^{4}$ used Plaster of Paris (Varsity Flask Hanau Engineering Company NY) and Nelson et $\mathrm{al}^{8}$ used gypsum for investing. In the present study, artificial stone (Pars Dandan, Tehran, Iran) was used.

Basso et $\mathrm{al}^{2}$ used a lucitone 550 acryl resin (Dentsply, York, PA) with the compression molding technique. ${ }^{2}$ Keenan et $\mathrm{al}^{4}$ employed trevalon (Dentsply) for two groups, acron (Dentsply) for the third and microbase (Dentsply) for

\begin{tabular}{|c|c|c|}
\hline & Number of occlusal contacts & $C V$ \\
\hline Before processing & $10.9 \pm 2.4$ & 22.0 \\
\hline After processing & $6.3 \pm 3.1$ & 47.6 \\
\hline
\end{tabular}

\begin{tabular}{|c|c|c|c|}
\hline \multirow{2}{*}{$\begin{array}{l}\text { Changes of occlusal } \\
\text { contacts }\end{array}$} & \multicolumn{2}{|c|}{ OVD changes } & \multirow[t]{2}{*}{ Total } \\
\hline & $<2 m m$ & $\geq 2 \mathrm{~mm}$ & \\
\hline$<6$ & $23(52.3 \%)$ & $21(47.7 \%)$ & $44(100 \%)$ \\
\hline$\geq 6$ & 2(11.1\%) & $16(88.9 \%)$ & $18(100 \%)$ \\
\hline
\end{tabular}

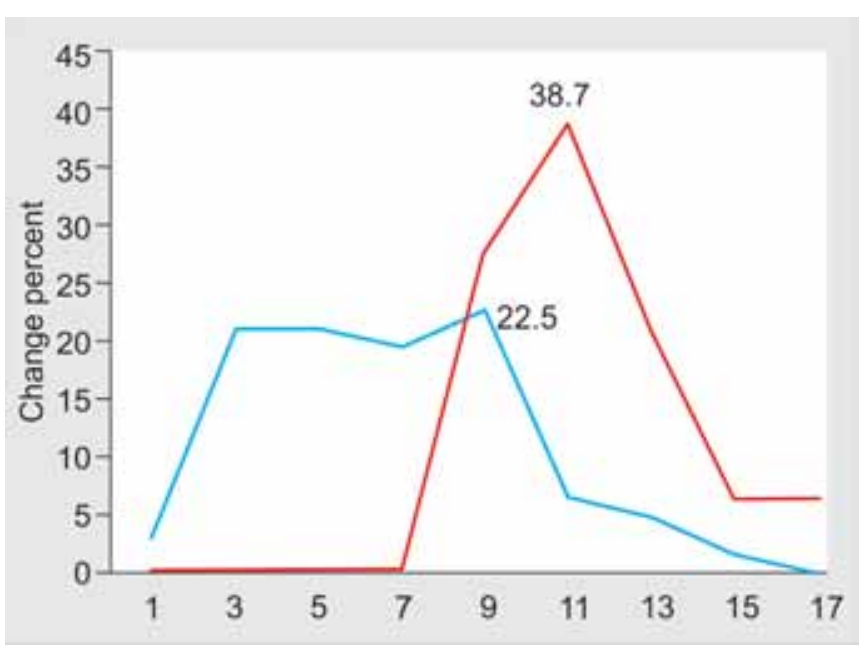

Fig. 3: The distribution of the complete dentures according to the percent of occlusal contact changes before and after processing. The blue line demonstrates the variation coefficient of the complete dentures before processing and the red line shows the same values after processing the fourth group. They used compression and injection molding for the first and the other three study groups, respectively. Swards et $\mathrm{al}^{6}$ used Hy-Pro Lucitone (Dentsply) with compression molding for the first group. In the second group, they used 199 Lucitone (Dentsply) with the injection molding method. PERform CG (Anrico, Heber Springs, AR) was used with the fluid resin technique for the third group and Acron MC (GC Lab Technologies, Lockport, IL) with compression molding was employed for the fourth group. Nogueira et $\mathrm{al}^{7}$ used 199 Lucitone acryl (Dentsply, York, PA) and compression molding for the first group and injection molding for the second group. Nelson et $\mathrm{al}^{8}$ employed Lucitone and compression molding. Strohaver ${ }^{9}$ used Microlon LW (Hygienic Corp, Akron, OH) with compression molding for one group and injection molding for the other. In the present study, Selectaplus H/Trevalon (Dentsply) was incorporated for all of the complete dentures.

Trial closure to remove the excess acryl during compression was only used in the studies of Basso et $\mathrm{al}^{2}$ (once) and Keenan et $\mathrm{al}^{4}$ (twice). In the present study, trial closure was repeated for each denture until no excess acryl was observed.

Strohaver ${ }^{9}$ reported that a water bath was applied but the authors did not mention its type. In the study of Basso et $\mathrm{al}^{2}$ and Nogueira et al, ${ }^{7}$ a long cure water bath was used. Keenan et $\mathrm{al}^{4}$ used a hot air oven for two groups and a microwave for the other two groups. Swards et al ${ }^{6}$ employed heat polymerization (without mentioning its type) for 9 hours at $73.8^{\circ} \mathrm{C}$ for two groups, for 0.5 hours at $45^{\circ} \mathrm{C}$ for the third group and a microwave for the fourth. Nelson et $\mathrm{al}^{8}$ used a long cure water bath for one group and a microwave for the other.

Basso et $\mathrm{al}^{2}$ compared lingualized and balanced occlusion on the OVD and found no significant differences. Campos et $\mathrm{al}^{3}$ compared occlusal and OVD changes in complete removable dentures with the application of pack and press and injection molding. These authors reported that injection molding is associated with a smaller loss in the occlusal contacts and no change in the OVD. Keenan et $\mathrm{al}^{4}$ compared the occlusal and vertical height stability of complete removable dentures prepared by different combinations of injection/compression molding and microwave/hot air oven heating and found no significant differences between the groups after 28 days. Swards et $\mathrm{al}^{6}$ reported that the OVDs of dentures prepared with compression molding and heat polymerization were significantly changed after processing. All groups showed significant differences in the vertical height after 48 hours, but the changes were smaller than with the remounting process. Time was suggested as an influential factor in the 
dislocation of the incisal pin. In the study of Nogueira et $\mathrm{al}^{7}$ vertical height was significantly less in dentures prepared with compression molding compared with those prepared with injection molding. Nelson et $\mathrm{al}^{8}$ reported that the OVD stability was significantly less in dentures processed using a water bath compared with those processed using a microwave. Strohaver ${ }^{9}$ suggested that compression molding is associated with more stability compared with injection molding.

The OVD stability is much higher with the application of the long cure water bath than with the fast-processing technique used in the present study. ${ }^{2,7-9}$ The fast-processing technique is the most commonly used processing method because it is both simpler and less time-consuming. However, several problems need to be resolved when employing this method, specifically, the occlusal errors that occurred with the application of this technique and the loss of the occlusal morphology. The vertical height is increased less with the injection molding technique than with compression molding. ${ }^{3,4,6,7,9}$ Compression molding is the most common molding method and was used in the present study. The decrease in the number of the occlusal contacts and the increase in the vertical dimension of occlusion may be related to the heating duration and intensity, to compression during molding or to the lack of uniform thickness in the flanges.

\section{CONCLUSION}

Considering the limitations of the present study (master casting of different patients without uniform dimensions in the flanges and various inter-ridge distances), the fastprocessing technique in the water bath was concluded to decrease the number of occlusal contacts and to increase the OVD of the complete dentures. The occlusion may be corrected by selective reduction of the artificial teeth surface, which has its own disadvantages, including the deterioration of the occlusal pattern of the artificial teeth. On the contrary, failure to correct the occlusion may jeopardize the retention and resistance of dentures and result in resorption of the residual ridge. Therefore, the fastprocessing technique is not recommended for use with the water bath. Further studies are needed to assess the influence of various factors.

\section{CLINICAL SIGNIFICANCES}

Due to the decreasing of occlusal contacts and increasing of the OVD of complete dentures, more time is needed to adjust the occlusal errors and restoring the occlusal vertical dimension. Therefore, the fast-processing technique has a negative effect on the morphologic pattern of artificial teeth of complete dentures and thus should be used carefully.

\section{REFERENCES}

1. Jacob F, Zarb GA, Bolender $\mathrm{CH}$. Waxing and processing the dentures, their insertion, and follow-up. In: Zarb GA, Bolender CL, Eckert SE, Fenton AH, Jacob RF, MericskeStern R. Prosthodontic treatment for edentulous patients: Complete dentures and implant-supported prostheses (12th ed). St Louis: Mosby 2004;389-425.

2. Basso MF, Nogueira SS, Arioli-Filho JN. Comparison of the occlusal vertical dimension after processing complete dentures made with linqualized balanced occlusion and conventional balanced occlusion. J Prosthet Dent 2006;96:200-04.

3. Campos MS, Cavalcanti BN, Cunha VP. Occlusal changes in complete dentures processed by pack-and-press and injectionpressing techniques. Eur J Prosthodont Restor Dent 2005;13: 78-80.

4. Keenan PL, Radford DR, Clark RK. Dimensional change in complete dentures fabricated by injection molding and microwave processing. J Prosthet Dent 2003;89:37-44.

5. Barbosa DB, Compagnoni MA, Leles CR. Changes in occlusal vertical dimension in microwave processing of complete dentures. Braz Dent J 2002;13:197-200.

6. Swords RL, Latta GH, Wicks RA, Huget EF. Periodic evaluation of the occlusal vertical dimension of maxillary dentures from the wax trial denture through 48 hours after polymerization. J Prosthodont 2000;9:189-94.

7. Nogueira SS, Ogle RE, Davis EL. Comparison of accuracy between compression- and injection-molding complete dentures. J Prosthet Dent 1999; 82:291-300.

8. Nelson MW, Kotwal KR, Sevedge SR. Changes in vertical dimension of occlusion in conventional and microwave processing of complete dentures. J Prosthet Dent 1991;65: 306-08.

9. Strohaver RA. Comparison of changes in vertical dimension between compression and injection molding complete dentures. J Prosthet Dent 1989;62:716-18.

10. Lu EY, Zhang FQ, Chen XJ, Chen JJ, Wang CT. A threedimensional analysis of measuring occlusal contacts of complete denture. Shanghai Kou Qiang Yi Xue 2006;15:623-26.

11. Kenji O, Shigezo H, Iwao H. A clinical application of the T-Scan II system-usefulness for evaluating occlusal contacts of complete denture wearers. Kokubyo Gakkai Zasshi 2002;69:277-84.

12. Ifteni G, Burlui V, Olaru C, Axinia D, Bahrim D. The terminal occlusal contacts of the edentulous patient with a complete denture. Rev Med Chir Soc Med Nat Iasi 1999;103:195-97.

13. Kumagai H, Watanabe T, Kobayashi K, Suzuki T, Nagao M, Nikawa $\mathrm{H}$, et al. Incidence of occlusal contacts with complete dentures during mastication using a 6-channel telemetry system: Preliminary measurements. J Oral Rehabil 1999;26:918-22.

14. Boening KW, Walter MH. Computer-aided evaluation of occlusal load in complete dentures. J Prosthet Dent 1992;67: 339-44.

15. Wilson J, Rees JS. Comparison of interocclusal contacts registered intraorally and after a remount procedure in complete denture patients. Eur J Prosthodont Restor Dent 2006;14: 146-50. 
16. Holt JE. Research on remounting procedures. J Prosthet Dent 1977;38:338-41.

17. Firtell DN, Finzen FC, Holmes JB. The effect of clinical remount procedures on the comfort and success of complete dentures. J Prosthet Dent 1987;57:53-57.

18. Landa JS. Troubleshooting in complete denture prosthesis (Part 4). Proper adjustment procedures. J Prosthet Dent 1960;10: 490-95.

19. Schuyler $\mathrm{CH}$. Fundamental principles in the correction of occlusal disharmony, natural and artificial. Contact Intraocul Lens Med J 1967;12:21-24 contd.

20. Rudd KD. Processing complete dentures. Dent Clin North Am 1996;40:121-49.

21. Anusavice KJ. Philips' science of dental materials (12th ed). China: WB Saunders: Elsevier 2003;722-30.

22. Atashrazm P, Dashti MH. The prevalence of occlusal disharmony and its associated causes in complete dentures. J Contemp Dent Pract Sep 1 2009;10(5):E041-48.

\section{ABOUT THE AUTHORS}

\section{Parsa Atashrazm}

\section{(Corresponding Author)}

Associate Professor, Department of Prosthodontics, Dental Branch Islamic Azad University, Tehran, Iran, Phone: +982122255745 Fax: +982122264236, e-mail: p_atashrazm@sbmu.ac.ir

\section{Leila Zamani Alavijeh}

Postgraduate Student, Department of Prosthodontics, Islamic Azad University, Dental Branch, Tehran, Iran

\section{Maryam Sadat Sadrzadeh Afshar}

Instructor, Department of Prosthodontics, Islamic Azad University Dental Branch, Tehran, Iran 